\title{
Dual trigger improves response to ovarian stimulation and ICSI outcomes in patients with a previous r-hCG triggered ICSI cycle
}

\author{
Amanda Souza Setti ${ }^{1,2}$, Luis Guilherme Louzada Maldonado ${ }^{1}$, Daniela Paes de Almeida Ferreira Braga ${ }^{1,2}$, \\ Assumpto Iaconelli Jr. ${ }^{1,2}$, Edson Borges Jr. ${ }^{1,2}$ \\ ${ }^{1}$ Fertility Medical Group, São Paulo, SP, Brazil \\ ${ }^{2}$ Instituto Sapientiae - Centro de Estudos e Pesquisa em Reprodução Humana Assistida, São Paulo, SP, Brazil
}

\begin{abstract}
Objective: To evaluate if ovarian response to controlled ovarian stimulation (COS) and intracytoplasmic sperm injection (ICSI) outcomes are improved by the use of dual trigger (gonadotropin-releasing hormone $(\mathrm{GnRH})$ agonists plus recombinant human chorionic gonadotropin ( $r-h C G)$ ) in patients with previous cycles triggered with r-hCG.

Methods: This case-control study included 88 matched cycles performed in 88 patients, which had the first ICSI cycle triggered with r-hCG $(n=44)$, and the following ICSI cycle with dual trigger $(n=44)$. We compared the cycle outcomes between the groups. In a second case-control within-subject analyses, we compared the ICSI outcomes between patients which had the first ICSI cycle triggered with r-hCG only $(n=18)$, and the following ICSI cycle with dual trigger $(n=18)$ or $r$-hCG only $(n=18)$.

Results: Upon investigating repeated cycles ( $r-\mathrm{hCG}$ only vs. dual trigger), we found higher oocyte yield and mature oocyte rates, lower immature oocyte rates, higher fertilization rates, and higher blastocyst development rates; and higher rates of cycles with embryos transferred and implantation in the dual trigger cycle.

Conclusions: The dual trigger regimen is a more effective approach than r-hCG trigger in patients with a previous r-hCG triggered ICSI cycle, yielding improved response to COS, and better laboratorial and clinical outcomes.
\end{abstract}

Keywords: ICSI, oocyte maturation, ovarian stimulation, implantation

\section{INTRODUCTION}

For assisted reproductive technology, we usually trigger final follicular maturation administering recombinant human Chorionic Gonadotropin ( $\mathrm{r}-\mathrm{hCG}$ ) as a surrogate for the natural LH surge. More recently, gonadotropin-releasing hormone $(\mathrm{GnRH})$ agonists have been applied to that end, primarily in high responder patients, to prevent early onset ovarian hyperstimulation syndrome (OHSS), due to the induction of more physiologic $\mathrm{LH}$ and $\mathrm{FSH}$ releases (Griesinger et al., 2006; Humaidan et al., 2011).

The combination of a GnRH agonist and hCG to trigger final oocyte maturation is called "dual trigger" (Shapiro et al., 2008). The use of dual trigger to induce an endogenous LH surge has been explored in different scenarios. In patients with a history of immature oocytes, it seems to improve the incidence of mature oocytes (Griffin et al., 2014). The benefits of using a dual trigger have also been demonstrated in patients with repetitive immature oocytes retrieved, and empty follicle syndrome (Castillo et al., 2013). Zhang et al. (2017) reported a significantly higher number of retrieved oocytes, a higher percentage of mature oocytes, and better oocyte yield in dual trigger groups, compared with the conventional hCG trigger group. In addition, there were significantly improved pregnancy outcomes in normal responders (Lin et al., 2013), in women with diminished ovarian reserve (Lin et al., 2019), and in patients with a history of poor fertilization (Pereira et al., 2016; Elias et al., 2017). However, the advantage of using dual trigger in patients who previously failed an intracytoplasmic sperm injection (ICSI) cycle is still unclear.

The goal of this study was to compare ovarian response to controlled ovarian stimulation (COS) and ICSI outcomes among patients in whom final follicular maturation was achieved with the administration of dual trigger or standard r-hCG trigger.

\section{MATERIALS AND METHODS}

\section{Experimental design, patients, inclusion and ex-} clusion criteria

This case-control study included data obtained via chart review from 88 cycles matched by female age, performed in 88 patients undergoing ICSI between January and March 2019, in a private university-affiliated IVF center. The inclusion criteria were as follows: couples with primary infertility undergoing their first ICSI cycle, because of male factor infertility, whom had fresh embryo transfer on day 5 of embryo development.

We used rFSH for COS in all patients, and we achieved pituitary suppression with $\mathrm{GnRH}$ antagonist (cetrorelix acetate). We achieved final follicular maturation with the administration of GnRH agonist and r-hCG in the Dual Trigger Group $(n=44)$, and r-hCG in r-hCG Only Trigger Group $(n=44)$.

Ovarian response to COS and ICSI outcomes were compared between the groups. Primary endpoints were the number and maturity of retrieved oocytes.

In a subsequent analysis, we ran a retrospective case-control within-subject analysis, to compare the response to COS and ICSI outcomes in patients who had the first ICSI cycle triggered with r-hCG only ( $r$-hCG Group, $\mathrm{n}=18$ ), and the following ICSI cycle triggered with $\mathrm{GnRH}$ agonist and r-hCG (Dual Trigger Group, $n=18$ ). We also compared the cycles' outcomes of patients that had two consecutive ICSI cycles triggered with $\mathrm{r}-\mathrm{hCG}$ only $\left(\mathrm{r}-\mathrm{hCG} 1^{\text {st }}\right.$ Cycle Group, $n=18$ and r-hCG $2^{\text {nd }}$ Cycle Group, $n=18$ ) (Figure 1). For that, we matched the first ICSI cycles with those from the previous analysis, in order to verify if patients presenting with similar characteristics, who had their previous cycles triggered with r-hCG only would also benefit from a following cycle triggered with r-hCG only. The cycles were matched based on female age ( $\pm 1 \mathrm{y}$-old), mature follicular rate $( \pm 5 \%)$, number of retrieved oocytes $( \pm 2)$, mature oocyte rate $( \pm 5 \%)$, blastocyst development rate $( \pm 5 \%)$.

All patients signed a written informed consent form. The local Institutional Review Board approved the study. 
$1^{\text {st }}$ analysis

$2^{\text {nd }}$ analysis

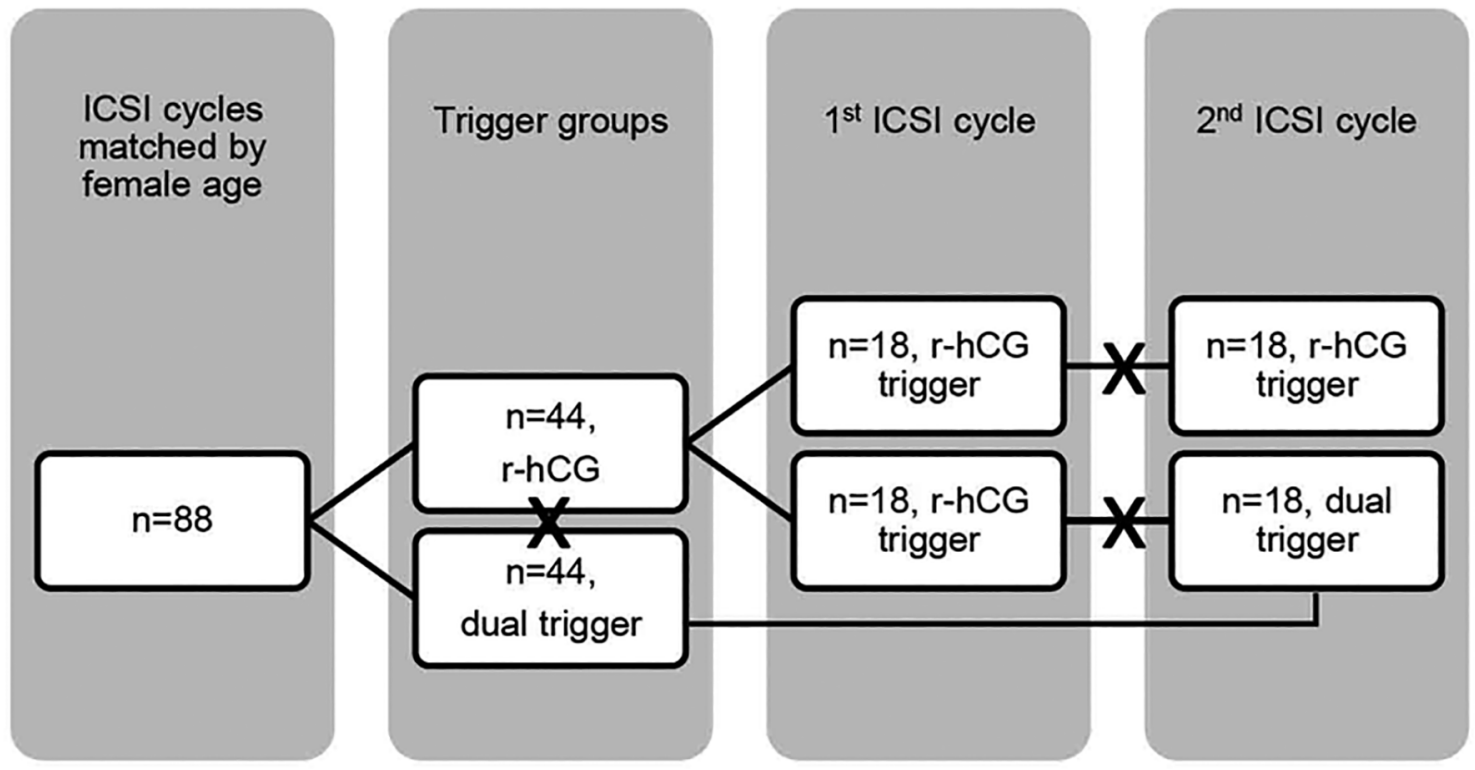

Figure 1. Study design Note: " $X$ " marks the comparisons.

\section{Controlled ovarian stimulation}

On the third day of the cycle, we started controlled ovarian stimulation by the administration of r-FSH (Gonal-F, Serono, Geneva, Switzerland) daily doses. When at least one follicle $\geq 14 \mathrm{~mm}$ was seen, pituitary blockage was performed using gonadotropin-releasing hormone $(\mathrm{GnRH})$ antagonist (GnRHa, Cetrotide ${ }^{\circledR}$; Merck KGaA, Darmstadt, Germany). When three or more follicles attained a mean diameter of $\geq 17 \mathrm{~mm}$ and there were adequate serum estradiol levels, we triggered the final follicular maturation with the administration of a GnRH agonist (triptorelin $0.1 \mathrm{mg}$, Gonapeptyl; Ferring $\mathrm{GmbH}$, Kiel, Alemanha) and r-hCG $\left(250 \mu \mathrm{g}\right.$, Ovidrel ${ }^{\circledR}$, Merck KGaA, Geneva, Switzerland) in the Dual Trigger Group or r-hCG (Ovidrel ${ }^{\circledR}$ ) in the r-hCG-only Trigger Group. We retrieved the oocytes 35 hours later.

\section{Intracytoplasmic sperm injection, embryo cul- ture and transfer}

We performed intracytoplasmic sperm injection according to Palermo et al. (1992). We cultured the embryos in $50-\mu \mathrm{L}$ drops of culture medium (Global $\AA$, LifeGlobal, Guilford, USA), covered with paraffin oil, in a humidified atmosphere, under $6 \% \mathrm{CO}_{2}$ at $37^{\circ} \mathrm{C}$, for five days.

The blastocyst development rate was defined as the number of embryos that reached blastocyst stage at day five by the number of 2PN embryos in each cycle. On day 5 , we transferred one to two embryos per patient, depending on maternal age and embryo quality, using a soft catheter under transabdominal ultrasound guidance.

Clinical follow-up

Clinical pregnancy was confirmed upon detection of at least one intrauterine gestational sac with fetal heartbeat at 6-8 weeks of gestation. We calculated the pregnancy rates per transfer. Miscarriage was defined as pregnancy loss before 20 weeks of gestation.

\section{Data analysis and statistics}

We compared the demographics between the groups using the Student's t-test. Follicles with a mean diameter of $\geq 17 \mathrm{~mm}$ on the trigger day were considered mature. The number of mature follicles per total number of follicles on the trigger day.

We compared the ICSI outcomes between the groups using general linear models (GLM) followed by Bonferroni post hoc, adjusted for paternal age, as this variable was significantly different between the groups. The oocyte and embryo utilization rates were calculated as the sum of transferred and frozen embryos per retrieved oocytes or fertilized oocytes, respectively, and were compared between the groups using GLM, followed by Bonferroni post hoc, adjusted for paternal age. The main outcome measure was the ovarian response to COS.

Data are expressed as mean \pm standard deviation and $p$-values. $P$-value was significant at $5 \%(<0.05)$. We ran the analysis using SPSS Statistics 21 (IBM, New York, New York, USA).

\section{RESULTS}

Table 1 describes the patients' demographics, response to COS and laboratorial ICSI outcomes. The Dual Trigger Group had a significantly higher number of retrieved oocytes, oocyte yield, and lower incidence of metaphase I compared to the r-hCG Trigger Group. There were no significant differences for other variables.

The oocyte utilization rate $(26.11 \% \pm 4.10$ vs. $27.37 \% \pm 4.10, p=0.829)$ and the embryo utilization rate $(48.45 \pm 5.34$ vs. $60.75 \pm 6.09, p=0.136)$ were similar between the Dual Trigger Group and the r-hCG Trigger Group, respectively.

There was a higher number of cycles with embryo transferred in the Dual Trigger Group, compared to the r-hCG Trigger Group 78.57\% (33/44) vs. 57.14\% (24/44), $p=0.035$. The groups had similar rates of implantation 


\begin{tabular}{|c|c|c|c|}
\hline Variables & Dual trigger $(n=44)$ & r-hCG trigger $(n=44)$ & p-value \\
\hline Female age (years) & $37.27 \pm 3.94$ & $37.91 \pm 3.80$ & 0.443 \\
\hline Male age (years) & $39.61 \pm 4.54$ & $37.18 \pm 5.08$ & 0.028 \\
\hline Female BMI & $24.83 \pm 3.11$ & $24.51 \pm 3.90$ & 0.682 \\
\hline FSH dose (IU) & $2685.26 \pm 829.53$ & $2519.58 \pm 877.67$ & 0.397 \\
\hline Length of stimulation (days) & $10.24 \pm 1.37$ & $10.92 \pm 1.71$ & 0.082 \\
\hline Length of pituitary suppression (days) & $4.49 \pm 1.03$ & $4.64 \pm 1.19$ & 0.584 \\
\hline Total number of follicles on trigger day & $10.64 \pm 6.53$ & $8.43 \pm 6.59$ & 0.119 \\
\hline Mature follicular rate $(\%)$ & $36.12 \pm 17.38$ & $43.73 \pm 26.10$ & 0.210 \\
\hline Estradiol level (pg/mL) & $1685.77 \pm 992.52$ & $1188.57 \pm 1009.53$ & 0.155 \\
\hline Number of retrieved oocytes & $7.89 \pm 5.31$ & $5.30 \pm 5.07$ & 0.022 \\
\hline Oocyte yield (\%) & $72.51 \pm 24.77$ & $55.10 \pm 30.50$ & 0.005 \\
\hline MII oocyte rate (\%) & $70.33 \pm 26.87$ & $63.17 \pm 30.29$ & 0.266 \\
\hline MI oocyte rate $(\%)$ & $6.50 \pm 2.14$ & $14.53 \pm 20.92$ & 0.044 \\
\hline PI oocyte rate (\%) & $17.45 \pm 22.90$ & $15.70 \pm 24.16$ & 0.741 \\
\hline Fertilization rate $(\%)$ & $84.27 \pm 29.63$ & $77.58 \pm 35.03$ & 0.373 \\
\hline Blastocyst development rate (\%) & $29.98 \pm 39.72$ & $30.66 \pm 30.69$ & 0.948 \\
\hline
\end{tabular}

Note: values are mean \pm standard deviation, unless otherwise noted. COS - controlled ovarian stimulation, r-hCG recombinant human chorionic gonadotropin, BMI - body mass index, IU - international unit, MII - metaphase II, MI metaphase I, PI - prophase I.

(23.74 \pm 39.54 vs. $27.27 \pm 45.58, p=0.761)$, clinical pregnancy $(10 / 33,30.30 \%$ vs. $8 / 24,33.33, p=0.808)$, and miscarriage $(1 / 10,10.0 \%$ vs. $1 / 8,12.5 \%, p=0.999)$, respectively.

In the subsequent case-control within-subject analysis; upon investigating repeated cycles ( $\mathrm{r}$-hCG only $v s$. dual trigger), there were higher oocyte yield, higher mature oocyte rate, lower immature oocyte rate, higher fertilization rate, and higher blastocyst development rate in the Dual Trigger Group compared to the r-hCG Trigger Group (Table 2).

The oocyte utilization rate tended to be higher in the Dual Trigger Group compared to the hCG Trigger Group (32.31\% \pm 3.46 vs. $17.68 \% \pm 1.09, p=0.074$, respectively). The embryo utilization rate was significantly higher in the Dual Trigger Group compared to the hCG Trigger Group (59.04\% 2.06 vs. 38.59\% $\pm 1.87, p<0.001$, respectively).

A higher number of cycles with embryo transfer $(16 / 18,88.89 \%$ vs. $10 / 18,55.55 \%, p=0.026)$ and a higher implantation rate $(26.92 \% \pm 1.44$ vs. $10.00 \% \pm 1.00$, $p<0.001$ ) were seen in the Dual Trigger $2^{\text {nd }}$ Cycle Group compared to the r-hCG-only trigger $1^{\text {st }}$ Cycle Group, respectively. The groups had similar rates of clinical pregnancy $(7 / 16,43.75 \%$ vs. $1 / 10,10.00 \%, p=0.099)$, and miscarriage $(1 / 7,14.29 \%$ vs. $1 / 1,100 \%, p=0.250)$, respectively.

Upon investigating repeated cycles ( $r$-hCG only vs. $\mathrm{r}$-hCG only), there were lower immature oocyte rate and higher implantation rate in the r-hCG Trigger $2^{\text {nd }}$ Cycle Group compared to r-hCG Trigger $1^{\text {st }}$ Cycle Group (Table 3).

The oocyte utilization rate $(30.21 \% \pm 1.55$ vs. $31.75 \% \pm 1.70, p=0.975)$ and the embryo utilization rate $(64.03 \pm 2.56$ vs. $46.02 \% \pm 2.02, p=0.098)$ were similar between the r-hCG Trigger $1^{\text {st }}$ Cycle Group and the r-hCG Trigger $2^{\text {nd }}$ Cycle Group, respectively.
Similar number of cycles with embryo transferred (11/18, $61.11 \%$ vs. $13 / 18,72.22 \%, p=0.725)$ and clinical pregnancy rate $(3 / 11,27.27 \%$ vs. $0 / 13,0.00 \%, p=0.099)$ were seen between the groups. There were no miscarriages in both groups.

\section{DISCUSSION}

In this study, we found that patients with a previous r-hCG triggered ICSI cycle benefited from a dual trigger in the following ICSI cycle. Our results are in agreement with several previous studies reporting higher number of collected oocytes (Lin et al., 2013) and mature oocytes (Lin et al., 2013), higher top-quality embryo rate (Decleer et al., 2014), increased implantation (Lin et al., 2013) and pregnancy rates (Lin et al., 2013) in patients with a normal response to COS, when dual trigger was performed compared to conventional hCG trigger.

Previous studies performed in poor responder patients also demonstrated that dual trigger yields higher numbers of retrieved oocytes (Oliveira et al., 2016), more mature oocytes retrieved (Griffin et al., 2014; Zilberberg et al., 2015; Oliveira et al., 2016), higher fertilization (Oliveira et al., 2016) and top-quality embryo rates (Zilberberg et al., 2015), higher implantation (Oliveira et al., 2016) and pregnancy rates (Schachter et al., 2008), and higher livebirth rate (Oliveira et al., 2016).

Differences in the results of the aforementioned studies might be attributed to the $\mathrm{GnRH}$ analogue used for pituitary suppression, origin and dose of the hCG administered. In fact, it has been shown that GnRH agonist for trigger after the use of $\mathrm{GnRH}$ antagonist for pituitary suppression increases implantation rates (Schachter et al., 2008). This happens because, initially, the GnRH antagonist blocks endometrial $\mathrm{GnRH}$ receptors. After the administration of $\mathrm{GnRH}$ antagonist as trigger of final oocyte maturation, the $\mathrm{GnRH}$ antagonist is displaced from the endometrial receptors, improving endometrial receptivity (Schachter et al., 2008). 
Table 2. Descriptive analysis of patients' demographics, response to COS and laboratorial ICSI outcomes in repeated cycles.

\begin{tabular}{|l|c|c|c|}
\hline Variables & r-hCG Trigger (n=18) & Dual Trigger (n=18) & p-value \\
\hline Female age (years) & $37.83 \pm 0.77$ & $38.22 \pm 0.77$ & 0.721 \\
\hline Male age (years) & $39.06 \pm 0.93$ & $39.44 \pm 0.93$ & 0.767 \\
\hline Female BMI & $24.66 \pm 0.58$ & $25.33 \pm 0.58$ & 0.414 \\
\hline FSH dose (IU) & $2875.00 \pm 190.44$ & $2625.00 \pm 201.99$ & 0.368 \\
\hline Length of stimulation (days) & $10.41 \pm 1.33$ & $10.47 \pm 1.70$ & 0.911 \\
\hline Length of pituitary suppression (days) & $4.76 \pm 0.83$ & $4.53 \pm 1.23$ & 0.518 \\
\hline Total number of follicles on trigger day & $11.17 \pm 1.799$ & $10.56 \pm 1.80$ & 0.095 \\
\hline Mature follicular rate (\%) & $46.63 \pm 20.30$ & $34.54 \pm 21.23$ & 0.644 \\
\hline Estradiol level (pg/mL) & $1246.67 \pm 261.21$ & $1431.22 \pm 301.62$ & 0.772 \\
\hline Number of retrieved oocytes & $7.17 \pm 1.36$ & $7.72 \pm 1.36$ & $<0.001$ \\
\hline Oocyte yield (\%) & $60.83 \pm 3.18$ & $84.38 \pm 3.25$ & $<0.001$ \\
\hline MII oocyte rate (\%) & $59.50 \pm 2.44$ & $74.29 \pm 3.26$ & $<0.001$ \\
\hline MI oocyte rate (\%) & $18.22 \pm 2.63$ & $5.80 \pm 2.55$ & $<0.001$ \\
\hline PI oocyte rate (\%) & $16.11 \pm 1.34$ & $88.12 \pm 2.35$ & 0.027 \\
\hline Fertilization rate (\%) & $80.00 \pm 2.83$ & $41.67 \pm 2.63$ & $<0.001$ \\
\hline Blastocyst development rate (\%) & $18.21 \pm 1.14$ & & $<$ \\
\hline
\end{tabular}

Note: Retrospective case-control within-subject analysis performed to compare the response to COS and ICSI outcomes in patients who had the first ICSI cycle triggered with r-hCG only and the following ICSI cycle triggered with GnRH agonist and r-hCG. Values are mean \pm standard error, unless otherwise noted. COS - controlled ovarian stimulation, ICSI - intracytoplasmic sperm injection, r-hCG - recombinant human chorionic gonadotropin.

Table 3. Descriptive analysis of patients' demographics, response to COS and laboratorial ICSI outcomes in repeated cycles.

\begin{tabular}{|l|c|c|c|}
\hline Variables & r-hCG Trigger 1 $\mathbf{1}^{\text {st }}$ Cycle (n=18) & r-hCG Trigger 2nd Cycle (n=18) & p-value \\
\hline Female age (years) & $37.44 \pm 0.14$ & $37.44 \pm 0.14$ & $>0.999$ \\
\hline Male age (years) & $38.72 \pm 1.65$ & $39.11 \pm 1.65$ & 0.868 \\
\hline Female BMI & $24.72 \pm 0.95$ & $25.00 \pm 0.95$ & 0.833 \\
\hline FSH dose (IU) & $2620.59 \pm 154.62$ & $2841.67 \pm 150.26$ & 0.305 \\
\hline Length of stimulation (days) & $10.41 \pm 1.70$ & $10.94 \pm 1.75$ & $>0.999$ \\
\hline Length of pituitary suppression (days) & $4.88 \pm 1.17$ & $4.88 \pm 1.17$ & 0.468 \\
\hline Number of follicles & $10.00 \pm 2.44$ & $12.50 \pm 2.44$ & 0.242 \\
\hline Mature follicular rate (\%) & $44.97 \pm 26.90$ & $35.57 \pm 18.26$ & 0.927 \\
\hline Estradiol level (pg/mL) & $1520.31 \pm 227.97$ & $1556.69 \pm 322.40$ & 0.178 \\
\hline Number of retrieved oocytes & $6.00 \pm 1.87$ & $9.56 \pm 1.87$ & 0.190 \\
\hline Oocyte yield (\%) & $61.81 \pm 2.62$ & $72.23 \pm 2.62$ & 0.686 \\
\hline MII oocyte rate (\%) & $62.41 \pm 2.63$ & $65.54 \pm 2.63$ & 0.011 \\
\hline MI oocyte rate (\%) & $17.00 \pm 1.30$ & $12.72 \pm 1.08$ & 0.450 \\
\hline PI oocyte rate (\%) & $9.09 \pm 0.91$ & $10.00 \pm 0.79$ & 0.655 \\
\hline Fertilization rate (\%) & $66.25 \pm 2.88$ & $67.92 \pm 2.38$ & 0.145 \\
\hline Blastocyst development rate (\%) & $19.75 \pm 2.14$ & $34.51 \pm 1.79$ & \\
\hline
\end{tabular}

Note: Retrospective case-control within-subject analysis performed to compare the response to COS and ICSI outcomes in patients who had two consecutive ICSI cycles triggered with r-hCG only. Values are mean \pm standard error, unless otherwise noted. COS - controlled ovarian stimulation, ICSI - intracytoplasmic sperm injection, r-hCG - recombinant human chorionic gonadotropin. 
The use of dual trigger with GnRH agonist and hCG to induce final oocyte maturation combines the advantages of both hormone regimens with (i) the induction of a physiological release of FSH through the FSH peak induced by the GnRH agonist, and (ii) improving luteal phase recruitment (Shapiro et al., 2011). These issues will be discussed below.

The induction of an FSH peak by the GnRH agonist in the dual trigger, in addition to the LH surge, has been shown to increase the number of mature oocytes (Zeleznik et al., 1974; Richards et al., 1976; Lamb et al., 2011; Castillo et al., 2013; Griffin et al., 2014). FSH surge is associated with oocyte maturation, expansion of cumulus cells and release of proteolytic enzymes involved in the ovulation process (Yding Andersen, 2002; Richards et al., 2005).

The GnRH-a trigger down-regulates the pituitary gland and reduces $\mathrm{LH}$ to a level that is insufficient for maintaining corpus luteum function (Shapiro \& Andersen, 2015). The GnRH triggering of final oocyte maturation is associated with unfavorable effects on endometrial receptivity that can happen due to a direct effect, dysfunctional corpus luteum, or early luteolysis. Several studies have showed smaller midluteal phase ovarian volume (Engmann et al., 2008; Garcia-Velasco et al., 2010), significantly lower luteal phase steroidal concentration and peptides (Engmann et al., 2008), and shorter duration of the luteal phase in non-supplemented cycles (Garcia-Velasco et al., 2010) using the GnRH agonist trigger. This evidence supports corpus luteum dysfunction after GnRHa trigger. One of the consequences of dysfunctional corpus luteum is a potential injurious impact on endometrial receptivity. In fact, significantly higher miscarriage rates and lower ongoing pregnancy rates after GnRHa trigger have been reported (Humaidan et al., 2005; Kolibianakis et al., 2005). On the other hand, hCG-trigger causes the production of endogenous progesterone that stimulates uterine changes for successful implantation.

The results here presented might provide another tool for the clinician to use in the trigger-regimen decision-making process. However, this is a retrospective study with its inherent limitations and bias. This study was limited by its small sample size.

In conclusion, the dual trigger regimen is a more effective approach than the r-hCG trigger in patients with a previous r-hCG triggered ICSI cycle, yielding improved ovarian response and ICSI outcomes.

\section{CONFLICT OF INTEREST}

The authors have no conflict of interest to declare.

\section{Corresponding author:}

Amanda Souza Setti

Fertility Medical Group

São Paulo - SP - Brazil

E-mail: amanda@sapientiae.org.br

\section{REFERENCES}

Castillo JC, Moreno J, Dolz M, Bonilla-Musoles F. Successful Pregnancy Following Dual Triggering Concept (rhCG $p$ GnRH agonist) in a Patient Showing Repetitive Inmature Oocytes and Empty Follicle Syndrome: Case Report. J Med Cases. 2013;4:221-6. DOI: 10.4021/jmc1055w

Decleer W, Osmanagaoglu K, Seynhave B, Kolibianakis S, Tarlatzis B, Devroey P. Comparison of hCG triggering versus hCG in combination with a GnRH agonist: a prospective randomized controlled trial. Facts Views Vis Obgyn. 2014;6:203-9. PMID: 25593695
Elias RT, Pereira N, Artusa L, Kelly AG, Pasternak M, Lekovich JP, Palermo GD, Rosenwaks Z. Combined GnRH-agonist and human chorionic gonadotropin trigger improves ICSI cycle outcomes in patients with history of poor fertilization. J Assist Reprod Genet. 2017;34:781-8. PMID: 28444614 DOI: $10.1007 / \mathrm{s} 10815-017-0917-3$

Engmann L, DiLuigi A, Schmidt D, Nulsen J, Maier D, Benadiva $C$. The use of gonadotropin-releasing hormone (GnRH) agonist to induce oocyte maturation after cotreatment with GnRH antagonist in high-risk patients undergoing in vitro fertilization prevents the risk of ovarian hyperstimulation syndrome: a prospective randomized controlled study. Fertil Steril. 2008;89:84-91. PMID: 17462639 DOI: 10.1016/j.fertnstert.2007.02.002

Garcia-Velasco JA, Motta L, López A, Mayoral M, Cerrillo $M$, Pacheco $A$. Low-dose human chorionic gonadotropin versus estradiol/progesterone luteal phase support in gonadotropin-releasing hormone agonist-triggered assisted reproductive technique cycles: understanding a new approach. Fertil Steril. 2010;94:2820-3. PMID: 20673892 DOI: $10.1016 /$ j.fertnstert.2010.06.035

Griesinger G, Diedrich K, Devroey P and Kolibianakis EM. $\mathrm{GnRH}$ agonist for triggering final oocyte maturation in the GnRH antagonist ovarian hyperstimulation protocol: a systematic review and meta-analysis. Hum Reprod Update. 2006;12:159-68. PMID: 16254001 DOI: 10.1093/humupd/dmi045

Griffin D, Feinn R, Engmann L, Nulsen J, Budinetz T, Benadiva C. Dual trigger with gonadotropin-releasing hormone agonist and standard dose human chorionic gonadotropin to improve oocyte maturity rates. Fertil Steril. 2014;102:405-9. PMID: 24842671 DOI: $10.1016 / j . f e r t n-$ stert.2014.04.028

Humaidan $\mathrm{P}$, Bredkjaer HE, Bungum L, Bungum M, Grøndahl $M L$, Westergaard L, Andersen CY. GnRH agonist (buserelin) or hCG for ovulation induction in GnRH antagonist IVF/ ICSI cycles: a prospective randomized study. Hum Reprod. 2005;20:1213-20. PMID: 15760966 DOI: 10.1093/humrep/deh765

Humaidan P, Kol S, Papanikolaou EG; Copenhagen GnRH Agonist Triggering Workshop Group. GnRH agonist for triggering of final oocyte maturation: time for a change of practice? Hum Reprod Update. 2011;17:510-24. PMID: 21450755 DOI: 10.1093/humupd/dmr008

Kolibianakis EM, Schultze-Mosgau A, Schroer A, van Steirteghem A, Devroey P, Diedrich K, Griesinger G. A lower ongoing pregnancy rate can be expected when GnRH agonist is used for triggering final oocyte maturation instead of HCG in patients undergoing IVF with GnRH antagonists. Hum Reprod. 2005;20:2887-92. PMID: 15979994 DOI: $10.1093 /$ humrep/dei150

Lamb JD, Shen S, McCulloch C, Jalalian L, Cedars MI, Rosen MP. Follicle-stimulating hormone administered at the time of human chorionic gonadotropin trigger improves oocyte developmental competence in in vitro fertilization cycles: a randomized, double-blind, placebo-controlled trial. Fertil Steril. 2011;95:1655-60. PMID: 21315341 DOI: 10.1016/j.fertnstert.2011.01.019 
Lin $\mathrm{MH}$, Wu FS, Lee RK, Li SH, Lin SY, Hwu YM. Dual trigger with combination of gonadotropin-releasing hormone agonist and human chorionic gonadotropin significantly improves the live-birth rate for normal responders in GnRH-antagonist cycles. Fertil Steril. 2013;100:1296-302. PMID: 23993928 DOI: 10.1016/j.fertnstert.2013.07.1976

Lin $\mathrm{MH}$, Wu FS, Hwu YM, Lee RK, Li RS, Li SH. Dual trigger with gonadotropin releasing hormone agonist and human chorionic gonadotropin significantly improves live birth rate for women with diminished ovarian reserve. Reprod Biol Endocrinol. 2019;17:7. PMID: 30609935 DOI: 10.1186/s12958-018-0451-x

Oliveira SA, Calsavara VF, Cortés GC. Final Oocyte Maturation in Assisted Reproduction with Human Chorionic Gonadotropin and Gonadotropin-releasing Hormone agonist (Dual Trigger). JBRA Assist Reprod. 2016;20:246-50. PMID: 28050961 DOI: 10.5935/1518-0557.20160047

Palermo G, Joris H, Devroey P, Van Steirteghem AC. Pregnancies after intracytoplasmic injection of single spermatozoon into an oocyte. Lancet. 1992;340:17-8. PMID: 1351601 DOI: $10.1016 / 0140-6736(92) 92425-F$

Pereira N, Elias RT, Neri QV, Gerber RS, Lekovich JP, Palermo GD, Rosenwaks Z. Adjuvant gonadotrophin-releasing hormone agonist trigger with human chorionic gonadotrophin to enhance ooplasmic maturity. Reprod Biomed Online. 2016;33:568-74. PMID: 27567429 DOI: 10.1016/j. rbmo.2016.08.009

Richards JS, Ireland JJ, Rao MC, Bernath GA, Midgley AR Jr, Reichert LE Jr. Ovarian follicular development in the rat: hormone receptor regulation by estradiol, follicle stimulating hormone and luteinizing hormone. Endocrinology. 1976;99:1562-70. PMID: 187412 DOI: 10.1210/endo-996-1562

Richards JS, Hernandez-Gonzalez I, Gonzalez-Robayna I, Teuling E, Lo Y, Boerboom D, Falender AE, Doyle KH, LeBaron RG, Thompson V, Sandy JD. Regulated expression of ADAMTS family members in follicles and cumulus oocyte complexes: evidence for specific and redundant patterns during ovulation. Biol Reprod. 2005;72:1241-55. PMID: 15659705 DOI: $10.1095 /$ biolreprod.104.038083
Schachter M, Friedler S, Ron-El R, Zimmerman AL, Strassburger $D$, Bern $O$, Raziel $A$. Can pregnancy rate be improved in gonadotropin-releasing hormone $(\mathrm{GnRH})$ antagonist cycles by administering GnRH agonist before oocyte retrieval? A prospective, randomized study. Fertil Steril. 2008;90:1087-93. PMID: 18023439 DOI: 10.1016/j.fertnstert.2007.07.1316

Shapiro BS, Daneshmand ST, Garner FC, Aguirre M, Thomas S. Gonadotropin-releasing hormone agonist combined with a reduced dose of human chorionic gonadotropin for final oocyte maturation in fresh autologous cycles of in vitro fertilization. Fertil Steril. 2008;90:231-3. PMID: 17981269 DOI: $10.1016 /$ j.fertnstert.2007.06.030

Shapiro BS, Daneshmand ST, Garner FC, Aguirre M, Hudson C. Comparison of "triggers" using leuprolide acetate alone or in combination with low-dose human chorionic gonadotropin. Fertil Steril. 2011;95:2715-7. PMID: 21550042 DOI: $10.1016 /$ j.fertnstert.2011.03.109

Shapiro BS, Andersen CY. Major drawbacks and additional benefits of agonist trigger--not ovarian hyperstimulation syndrome related. Fertil Steril. 2015;103:874-8. PMID: 25707333 DOI: 10.1016/j.fertnstert.2015.01.035

Yding Andersen C. Effect of FSH and its different isoforms on maturation of oocytes from pre-ovulatory follicles. Reprod Biomed Online. 2002;5:232-9. PMID: 12470520 DOI: $10.1016 / \mathrm{S} 1472-6483(10) 61826-3$

Zeleznik AJ, Midgley AR Jr, Reichert LE Jr. Granulosa cell maturation in the rat: increased binding of human chorionic gonadotropin following treatment with follicle-stimulating hormone in vivo. Endocrinology. 1974;95:818-25. PMID: 4368756 DOI: $10.1210 /$ endo-95-3-818

Zhang J, Wang Y, Mao X, Chen Q, Hong Q, Cai R, Zhang $S$, Kuang $Y$. Dual trigger of final oocyte maturation in poor ovarian responders undergoing IVF/ICSI cycles. Reprod Biomed Online. 2017;35:701-7. PMID: 28993105 DOI: 10.1016/j.rbmo.2017.09.002

Zilberberg E, Haas J, Dar S, Kedem A, Machtinger R, Orvieto R. Co-administration of $\mathrm{GnRH}$-agonist and hCG, for final oocyte maturation (double trigger), in patients with low proportion of mature oocytes. Gynecol Endocrinol. 2015;31:145-7. PMID: 25385007 DOI: $10.3109 / 09513590.2014 .978850$ 